\title{
Ecological Features of Cultivated Stands of Aquilaria malaccensis Lam. (Thymelaeaceae), a Vulnerable Tropical Tree Species in Assamese Homegardens
}

\author{
P. Saikia ${ }^{1}$ and M. L. Khan ${ }^{2}$ \\ ${ }^{1}$ Centre for Environmental Sciences, Central University of Jharkhand, Brambe, Ranchi, Jharkhand 835205, India \\ ${ }^{2}$ Department of Botany, Dr. Hari Singh Gour Central University, Sagar, Madhya Pradesh 470003, India
}

Correspondence should be addressed to P. Saikia; purabi.saikia@cuj.ac.in

Received 10 February 2014; Accepted 22 June 2014; Published 8 July 2014

Academic Editor: Sunil Nautiyal

Copyright (c) 2014 P. Saikia and M. L. Khan. This is an open access article distributed under the Creative Commons Attribution License, which permits unrestricted use, distribution, and reproduction in any medium, provided the original work is properly cited.

\begin{abstract}
Research was conducted in twenty-seven selected villages located in Jorhat and Golaghat districts of upper Assam, northeast India, for population estimation, quantitative ecological analysis, and evaluation of Aquilaria malaccensis (Thymelaeaceae). Vegetation sampling was done by quadrat method and A. malaccensis is the most dominant tree species in all twenty-seven different study sites of upper Assam contributing $10-54 \%$ of the total tree density with a mean of $34 \% \pm 2$. Density of the species varied from 6,236 individuals ha ${ }^{-1}$ to 429 individuals ha ${ }^{-1}$ with a mean of 1,609 individuals ha ${ }^{-1} \pm 217$, whereas frequency of occurrence is very high ranging from $93 \%$ to $100 \%$ with a mean of $98 \% \pm 0.53$ in different study sites. Distribution of $A$. malaccensis is found contagious in all twenty-six study sites on the basis of abundance to frequency ratio except in KBG, Golaghat, where its distribution is random with 0.04 abundance to frequency ratio. The widespread cultivation of A. malaccensis in upper Assam, northeast India, offers a potential ex situ reservoir for the future conservation and management of this threatened tree.
\end{abstract}

\section{Introduction}

Aquilaria malaccensis (Thymelaeaceae), locally known as "Sanchi" or "Agaru" in Assamese, is an evergreen tropical forest tree highly priced for its resin or agarwood. The species is valued in many cultures for centuries because of its distinctive fragrance and used extensively in incense, perfume, and traditional medicine. Natural populations of Agaru are distributed in south and southeast Asia and in India; it occurs mostly in foothills of northeastern region (Assam, Arunachal Pradesh, Nagaland, Meghalaya, Mizoram, Manipur, and Tripura) as well as West Bengal [1]. However, large scale harvesting of the species from natural population caused rapid depletion in the wild and is listed in Appendix II of the Convention on International Trade in Endangered Species of Wild Fauna and Flora (CITES) in 1994 [2]. The species is "vulnerable" globally according to the current IUCN red list [3]. On the other hand, Agaru is extensively cultivated in homegardens of upper Assam and contributes significantly up to $20 \%$ of the total annual income of the family with a mean of $4 \% \pm 0.46$ to the economy of the local people [4]. A good population stock of Agaru with highest density and frequency in homegardens of upper Assam was also reported by Saikia et al. [5]. Two distinct morphs of Agaru (Bhola Sanchi and Jati Sanchi) are cultivated in homegardens of upper Assam with different life form characteristics [6]. Bhola Sanchi is fast growing and less agarwood yielding than the other variant Jati Sanchi which is slow growing but high agarwood yielding and preferred for commercial cultivation. Nath and Saikia [7] also reported similar variations among the population of Agaru growing in homegarden and they identified three "races" from different areas of Assam. According to them, "variant I (RRLJ 2729)" is a medium sized tree with slender trunk, oblong-lanceolate leaves known as Bhola Sanchi; "variant II (RRLJ 2726)" is a large sized tree with obovate lanceolate leaves known as Sanchi; "variant III (RRLJ 2730)" is a much branched small to medium sized tree with lanceolate leaves known as Jati Sanchi. 
Quantitative inventories help in identifying species that are in different stages of vulnerability as well as various factors that influence the existing vegetation in any region $[8,9]$. Considering the scarcity of information on Agaru cultivation in homegardens of upper Assam, we attempted to study the ecological features, mainly, the quantitative characteristics of A. malaccensis Lam. in homegardens of upper Assam, northeast India.

\section{Materials and Methods}

2.1. Study Sites. The study was conducted in twenty-seven selected villages located in Jorhat and Golaghat districts of upper Assam, northeast India $\left(25^{\circ} 48^{\prime}\right.$ to $27^{\circ} 10^{\prime} \mathrm{N}$ and $93^{\circ} 17^{\prime}$ to $94^{\circ} 36^{\prime} \mathrm{E}$ ) (Figure 1). The site is surrounded by Sibsagar and Dibrugarh districts on the east, Nagaon and Karbi Anglong districts on the west, Lakhimpur and Sonitpur districts on the north, and the bordering state of Nagaland on the south. The total numbers of villages of Jorhat and Golaghat districts are 855 and 1089 with population density of 354 and 236 individuals per square kilometer, respectively [10]. The climate of the study area is classified as tropical type having distinct hot and humid summer $\left(34.0^{\circ} \mathrm{C}\right.$ during JuneJuly) and cool winter $\left(10.0^{\circ} \mathrm{C}\right.$ during December-January). The mean annual rainfall of Golaghat and Jorhat districts ranges between $1200 \mathrm{~mm}$ and $1900 \mathrm{~mm}$. The relative humidity remains very high throughout the year [11].

2.2. Vegetation Analysis. A total of twenty-seven different sites (16 from Golaghat and 11 from Jorhat district) of Jorhat and Golaghat districts of upper Assam, northeast India, were selected randomly from the pool of Agaru cultivating areas based on informal knowledge which roughly represented about 30\% of all Agaru growing areas in the site. A total of 135 homegardens (with a mean of 5 homegardens per site) were selected randomly and studied during 2007-2010 (Table 1). Vegetation was studied using quadrat method covering a minimum of $30 \%$ of the area in each homegarden. Random quadrats of $10 \mathrm{~m} \times 10 \mathrm{~m}$ size were used for trees and, within the same $10 \mathrm{~m} \times 10 \mathrm{~m}$ quadrat, one $5 \mathrm{~m} \times 5 \mathrm{~m}$ quadrat for shrubs and two $1 \mathrm{~m} \times 1 \mathrm{~m}$ quadrats for herbs were used in the studied homegardens. Diameter at breast height $(\mathrm{DBH})$ of all the individual trees was recorded during the study at $1.37 \mathrm{~m}$ above ground. Plant species were identified on the basis of vernacular names, published field inventories, floras, and consulting available herbaria of the region. Herbarium specimens were collected and deposited in the Department of Forestry, North Eastern Regional Institute of Science and Technology (Deemed University), Arunachal Pradesh.

2.3. Data Analysis. Quantitative analysis of vegetation was done following Misra [12]. Importance value index (IVI) was computed by summing up relative density, relative frequency, and relative dominance. The species richness was calculated by using the method "Margalef's index of richness" (Dmg) [13]:

$$
\operatorname{Dmg}=\frac{(S-1)}{\ln n}
$$
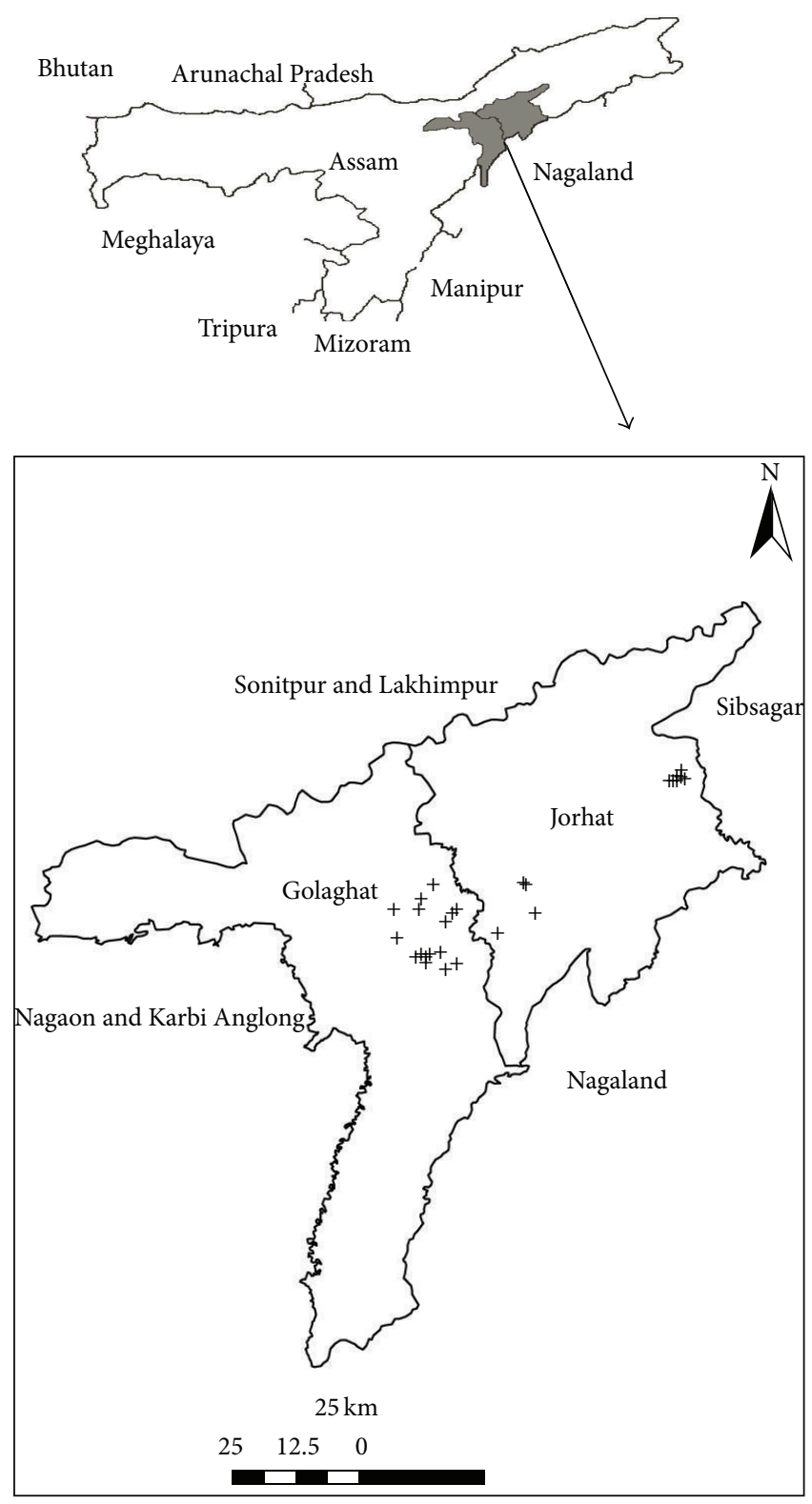

$+\quad$ Study area

$\square$ District boundary

FIGURE 1: Map of Golaghat and Jorhat districts of upper Assam, NE India, showing the locations of the study sites.

where $S=$ total number of species and $n=$ total number of individuals.

The Shannon-Wiener Diversity Index [14] was calculated from the IVI values using the formula given by Magurran [13]:

$$
H=-\sum_{i=1}^{s} p_{i} \ln p_{i}
$$

where $p_{i}$ is the proportion of the IVI of $i$ th species and the IVI of all the species $\left(n_{i} / N\right)$. 
TABLE 1: Number of studied homegardens and their mean size and number of associated species and dominant tree associate of different studied villages.

\begin{tabular}{|c|c|c|c|c|}
\hline Study sites (villages) & $\begin{array}{l}\text { HG studied } \\
\text { (number) }\end{array}$ & Mean HG area in ha $( \pm \mathrm{SE})$ & $\begin{array}{c}\text { Associated species } \\
\text { (numbers) }\end{array}$ & $\begin{array}{c}\text { Dominant tree } \\
\text { associate }\end{array}$ \\
\hline Buralikson (BS) & 2 & $0.17 \pm 0.04$ & 63 & Areca catechu $\mathrm{L}$. \\
\hline Bhumuraguri Missing Gaon (BMG) & 2 & $0.13 \pm 0.00$ & 62 & Mesua ferrea $\mathrm{L}$. \\
\hline Doloigaon (DG) & 2 & $0.11 \pm 0.02$ & 70 & Areca catechu $\mathrm{L}$. \\
\hline Dulakhariya (DK) & 2 & $0.16 \pm 0.11$ & 74 & Areca catechu $\mathrm{L}$. \\
\hline Elengi Thekela Gaon (ETG) & 5 & $0.24 \pm 0.03$ & 140 & Areca catechu $\mathrm{L}$. \\
\hline Ganakpukhuri (GP) & 5 & $0.16 \pm 0.03$ & 99 & Areca catechu $\mathrm{L}$. \\
\hline Gayan Gaon (GG) & 17 & $0.17 \pm 0.02$ & 187 & Areca catechu $\mathrm{L}$. \\
\hline Habungia Bamun Gaon (HBG) & 6 & $0.15 \pm 0.04$ & 117 & Areca catechu $\mathrm{L}$. \\
\hline Hatiyekhuwa (HK) & 2 & $0.09 \pm 0.01$ & 68 & Areca catechu $\mathrm{L}$. \\
\hline Hensowa Chengkola Gaon (HCG) & 4 & $0.23 \pm 0.06$ & 163 & Areca catechu L. \\
\hline Jalukonibari Thengal Gaon (JTG) & 6 & $0.21 \pm 0.03$ & 138 & Areca catechu $\mathrm{L}$. \\
\hline Junakinagar (JN) & 5 & $0.13 \pm 0.04$ & 110 & Areca catechu $\mathrm{L}$. \\
\hline Kabarugaon (KWG) & 14 & $0.16 \pm 0.01$ & 168 & Areca catechu L. \\
\hline Kakati Gaon (KG) & 4 & $0.15 \pm 0.03$ & 95 & Areca catechu $\mathrm{L}$. \\
\hline Kamarbandha Bamun Gaon (KBG) & 8 & $0.22 \pm 0.02$ & 159 & Areca catechu L. \\
\hline Kamarbandha Namsonia Gaon (KNG) & 8 & $0.17 \pm 0.02$ & 135 & Areca catechu L. \\
\hline Khumtai (KH) & 3 & $0.18 \pm 0.02$ & 120 & Areca catechu $\mathrm{L}$. \\
\hline Konhar Gaon (KHG) & 2 & $0.23 \pm 0.04$ & 67 & Areca catechu $\mathrm{L}$. \\
\hline Natun Gaon (NG) & 3 & $0.11 \pm 0.01$ & 91 & Areca catechu $\mathrm{L}$. \\
\hline Naharani Maj Gaon (NMG) & 2 & $0.17 \pm 0.10$ & 85 & Areca catechu $\mathrm{L}$. \\
\hline Pohusora (PS) & 4 & $0.12 \pm 0.01$ & 115 & Areca catechu $\mathrm{L}$. \\
\hline Pulibor (PB) & 8 & $0.21 \pm 0.02$ & 138 & Areca catechu $\mathrm{L}$. \\
\hline Puronimati Fatagaon (PFG) & 2 & $0.22 \pm 0.01$ & 90 & Areca catechu L. \\
\hline Sapani (SP) & 3 & $0.16 \pm 0.02$ & 108 & Areca catechu $\mathrm{L}$. \\
\hline Sialekhati (SK) & 3 & $0.18 \pm 0.02$ & 85 & Areca catechu $\mathrm{L}$. \\
\hline Sumoni Gaon (SMG) & 9 & $0.21 \pm 0.03$ & 163 & Areca catechu L. \\
\hline Sunari Gaon (SNG) & 4 & $0.09 \pm 0.02$ & 106 & Areca catechu L. \\
\hline
\end{tabular}

Concentration of dominance was assessed by Simpson's Index [15]:

$$
\mathrm{CD}=\sum_{i=1}^{s}\left(p_{i}\right)^{2}
$$

where $p_{i}$ is the same as for the Shannon-Wiener information function.

Evenness index was calculated from Shannon-Wiener Diversity Index using the formula

$$
E=\frac{H^{\prime}}{H^{\prime} \max },
$$

where $H^{\prime}$ is Shannon-Wiener Diversity Index and $H^{\prime} \max =$ $\ln S$ (where $S=$ total number of species).

Sørensen's similarity index was calculated using the formula given by Sorenson [16]:

$$
\text { Sørensen's similarity index }=\frac{2 C}{A+B} \times 100,
$$

where $C$ is the number of species common to two sites, $A$ is the total number of species in site $A$, and $B$ is the total number of species in site $B$.

The ratio of abundance to frequency was used to interpret the distribution pattern of the species [17]. The ratio of abundance to frequency indicates regular distribution if below 0.025 , random distribution if between 0.025 and 0.05 , and contagious distribution if $>0.05$ [18].

Statistical analysis (Standard error and $t$-test) has been done using statistical software like MS-Excel and ORIGIN.

\section{Results}

3.1. Ecological Features of A. malaccensis. A. malaccensis is the most dominant tree species in twenty-seven different study sites of upper Assam contributing 10-54\% of total tree density with a mean of $34 \% \pm 2$. Density of the species varied from 429 individuals $\mathrm{ha}^{-1}$ to 6,236 individuals $\mathrm{ha}^{-1}$ with a mean of 1,609 individuals ha ${ }^{-1} \pm 217$. Areca catech $u$ L. is the most dominant tree associate in twenty-six different study sites, 
TABLE 2: Community characteristics of different study sites of upper Assam, northeast India.

\begin{tabular}{|c|c|c|c|c|c|c|c|c|c|}
\hline $\begin{array}{l}\text { Study } \\
\text { sites }\end{array}$ & $\begin{array}{l}\text { Number of } \\
\text { families }\end{array}$ & $\begin{array}{l}\text { Number of } \\
\text { genera }\end{array}$ & $\begin{array}{l}\text { Species } \\
\text { richness }\end{array}$ & $\begin{array}{c}\text { Species } \\
\text { richness } \\
\text { index }\end{array}$ & $\begin{array}{c}\text { Density } \\
\text { (individuals } \mathrm{ha}^{-1} \text { ) }\end{array}$ & $\begin{array}{l}\text { Basal area } \\
\left(\mathrm{m}^{2} \mathrm{ha}^{-1}\right)\end{array}$ & $\begin{array}{c}\text { Diversity } \\
\left.\text { (Shannon's } H^{\prime}\right)\end{array}$ & $\mathrm{CD}$ & $\begin{array}{c}\text { Evenness } \\
\text { index }\end{array}$ \\
\hline BS & 39 & 57 & 64 & 4.27 & 4,410 & 1.08 & 3.01 & 0.06 & 0.91 \\
\hline $\mathrm{BMG}$ & 39 & 54 & 63 & 5.16 & 1,400 & 0.78 & 2.89 & 0.09 & 0.88 \\
\hline DG & 43 & 63 & 71 & 3.53 & 3,100 & 0.65 & 2.52 & 0.12 & 0.84 \\
\hline DK & 41 & 64 & 75 & 4.13 & 8,000 & 1.25 & 2.97 & 0.07 & 0.88 \\
\hline ETG & 64 & 113 & 141 & 8.22 & 3,625 & 2.48 & 3.37 & 0.07 & 0.82 \\
\hline GP & 52 & 85 & 100 & 7.72 & 3,197 & 1.74 & 3.44 & 0.05 & 0.86 \\
\hline GG & 73 & 150 & 188 & 9.49 & 3,332 & 2.11 & 3.58 & 0.06 & 0.82 \\
\hline HBG & 53 & 99 & 118 & 7.78 & 3,239 & 2.14 & 3.41 & 0.05 & 0.86 \\
\hline HK & 44 & 63 & 69 & 4.15 & 6,100 & 0.76 & 2.94 & 0.07 & 0.91 \\
\hline $\mathrm{HCG}$ & 67 & 136 & 164 & 8.30 & 5,341 & 3.09 & 3.56 & 0.05 & 0.86 \\
\hline JTG & 67 & 122 & 139 & 6.86 & 7,947 & 2.23 & 3.37 & 0.07 & 0.84 \\
\hline JN & 52 & 96 & 111 & 9.39 & 2,384 & 0.99 & 3.62 & 0.05 & 0.87 \\
\hline KWG & 71 & 141 & 169 & 10.54 & 4,370 & 2.29 & 3.66 & 0.06 & 0.82 \\
\hline KG & 53 & 83 & 96 & 6.20 & 3,710 & 0.93 & 3.22 & 0.06 & 0.86 \\
\hline KBG & 64 & 135 & 160 & 7.69 & 4,133 & 2.58 & 3.78 & 0.03 & 0.93 \\
\hline KNG & 60 & 116 & 136 & 7.60 & 3,531 & 3.81 & 3.10 & 0.10 & 0.77 \\
\hline $\mathrm{KH}$ & 52 & 103 & 121 & 6.47 & 10,381 & 1.63 & 3.26 & 0.08 & 0.84 \\
\hline KHG & 38 & 58 & 68 & 4.58 & 4,043 & 0.59 & 3.11 & 0.06 & 0.92 \\
\hline NG & 52 & 82 & 92 & 5.49 & 4,455 & 0.54 & 3.21 & 0.05 & 0.90 \\
\hline NMG & 52 & 78 & 86 & 5.21 & 13,418 & 0.99 & 3.22 & 0.07 & 0.88 \\
\hline PS & 58 & 95 & 116 & 5.27 & 6,200 & 1.05 & 3.10 & 0.08 & 0.86 \\
\hline PB & 67 & 119 & 139 & 9.23 & 2,731 & 2.19 & 3.50 & 0.07 & 0.83 \\
\hline PFG & 49 & 82 & 91 & 6.10 & 3,885 & 1.79 & 3.27 & 0.06 & 0.89 \\
\hline SP & 63 & 97 & 109 & 5.62 & 4,033 & 2.37 & 3.07 & 0.07 & 0.85 \\
\hline SK & 52 & 79 & 86 & 7.14 & 2,380 & 0.71 & 3.28 & 0.06 & 0.86 \\
\hline SMG & 65 & 132 & 164 & 7.77 & 5,205 & 4.40 & 3.29 & 0.07 & 0.79 \\
\hline SNG & 59 & 94 & 107 & 5.47 & 5,577 & 0.78 & 3.22 & 0.06 & 0.89 \\
\hline
\end{tabular}

whereas Mesua ferrea L. was the most dominant tree associate in BMG, Jorhat (Table 1). Similarly, A. malaccensis is the most frequent species followed by $A$. catechu and Bambusa pallida L. and frequency of occurrence of $A$. malaccensis ranged from $93 \%$ to $100 \%$ with a mean of $98 \% \pm 0.53$ in different study sites. $100 \%$ frequency was recorded at most of the study sites including BS, BMG, DG, DK, ETG, HK, HCG, JTG, KWG, KNG, KG, KBG, NG, NMG, PS, PFG, SMG, and SNG. Total basal cover was the highest $\left(1.25 \mathrm{~cm}^{2} \mathrm{ha}^{-1}\right)$ in NG, Jorhat, and the lowest $\left(0.34 \mathrm{~cm}^{2} \mathrm{ha}^{-1}\right)$ in JN, Golaghat. Among different sites, IVI of A. malaccensis ranged between 17.94 and 72.63 with a mean of $49.36 \pm 2.64$. On the other hand, abundance of the species in different study sites ranged from 4 to 62 with a mean of $16 \pm 2$ and it was the highest in NMG, Golaghat, and the lowest in KBG, Golaghat. Distribution of A. malaccensis is found contagious in all twenty-six study sites on the basis of abundance to frequency ratio except in KBG, Golaghat, where its distribution is random with 0.04 abundance to frequency ratio (Table 2).

3.2. Floristic Composition of Study Sites. A total of 323 plant species belonging to 241 genera under 95 families consisting of 106 (33\%) herbs, 61 (19\%) shrubs, and 156 (48\%) trees were recorded from twenty-seven study sites of upper Assam (Table 5). Although the number of documented trees was higher than that of herbs and shrubs, differences were not statistically significant. Overall data showed that family Euphorbiaceae had the highest number of species (15) followed by Moraceae (13) and Poaceae (12). Species richness was very high at all study sites ranging from 63 to 188 species with a mean of $113 \pm 7$, but the highest richness was recorded from GG, Jorhat, and the lowest from BMG, Jorhat (Table 3). A high variability in density of plant species was also noticed in different study sites. Tree density was the highest in NMG, Golaghat $\left(13,418\right.$ individuals $\left.\mathrm{ha}^{-1}\right)$, and the lowest in BMG, Jorhat (1,400 individuals $\left.\mathrm{ha}^{-1}\right)$. On the other hand, basal area of tree species was the highest in SMG, Golaghat $\left(4.40 \mathrm{~m}^{2} \mathrm{ha}^{-1}\right)$, and the lowest in NG, Jorhat $\left(0.54 \mathrm{~m}^{2} \mathrm{ha}^{-1}\right)$.

Peak richness index of tree species of 10.54 was recorded in KWG, Golaghat, whereas it was the lowest in DG, Jorhat (3.53). Concentration of dominance (Simpson's Index) and diversity (Shannon-Wiener Diversity Index) of tree species were showing reverse trend in study sites. Concentration of dominance (Simpson's Index) of tree species was the highest in DG, Jorhat (0.12), and the lowest in KBG, Golaghat 
TABLE 3: Ecological parameters of different populations of $A$. malaccensis $(>3.18 \mathrm{~cm} \mathrm{DBH})$.

\begin{tabular}{|c|c|c|c|c|c|c|c|}
\hline Study sites & Frequency (\%) & Density (individuals ha ${ }^{-1}$ ) & TBC $\left(\mathrm{cm}^{2} \mathrm{ha}^{-1}\right)$ & Abundance & IVI & Density share (\%) & $A / F$ ratio \\
\hline BS & 100 & 1,140 & 0.63 & 11 & 39.26 & 25.85 & 0.11 \\
\hline BMG & 100 & 709 & 0.64 & 7 & 72.63 & 50.65 & 0.07 \\
\hline DG & 100 & 1,686 & 0.65 & 17 & 72.45 & 54.38 & 0.17 \\
\hline DK & 100 & 3,273 & 0.93 & 33 & 59.12 & 40.91 & 0.33 \\
\hline ETG & 100 & 1,792 & 0.53 & 18 & 66.46 & 49.43 & 0.18 \\
\hline GP & 93 & 647 & 0.48 & 7 & 34.57 & 20.23 & 0.07 \\
\hline GG & 97 & 1,261 & 0.77 & 13 & 55.71 & 37.85 & 0.13 \\
\hline HBG & 96 & 896 & 0.50 & 9 & 42.42 & 27.67 & 0.10 \\
\hline HK & 100 & 1,700 & 0.57 & 17 & 37.19 & 27.87 & 0.17 \\
\hline HCG & 100 & 1,972 & 0.69 & 20 & 50.45 & 36.92 & 0.20 \\
\hline JTG & 100 & 1,700 & 0.57 & 17 & 35.07 & 21.39 & 0.17 \\
\hline JN & 94 & 858 & 0.34 & 9 & 50.56 & 35.99 & 0.10 \\
\hline KWG & 100 & 1,563 & 0.69 & 16 & 52.26 & 35.77 & 0.16 \\
\hline KNG & 100 & 1,793 & 0.70 & 18 & 68.41 & 50.79 & 0.18 \\
\hline $\mathrm{KG}$ & 100 & 965 & 0.75 & 10 & 42.44 & 26.01 & 0.10 \\
\hline KBG & 100 & 429 & 0.64 & 4 & 17.94 & 10.37 & 0.04 \\
\hline KHG & 93 & 1,121 & 0.77 & 12 & 43.50 & 27.74 & 0.13 \\
\hline $\mathrm{KH}$ & 94 & 1,756 & 0.68 & 19 & 30.05 & 16.92 & 0.20 \\
\hline NG & 100 & 927 & 1.25 & 9 & 35.22 & 20.82 & 0.09 \\
\hline NMG & 100 & 6,236 & 0.40 & 62 & 58.46 & 46.48 & 0.62 \\
\hline PS & 100 & 3,080 & 0.61 & 31 & 63.78 & 49.68 & 0.31 \\
\hline $\mathrm{PB}$ & 96 & 1,275 & 0.72 & 13 & 65.47 & 46.69 & 0.14 \\
\hline PFG & 100 & 1,254 & 0.69 & 13 & 46.64 & 32.28 & 0.13 \\
\hline SP & 93 & 1,467 & 1.08 & 16 & 50.54 & 36.36 & 0.17 \\
\hline SK & 95 & 905 & 0.42 & 10 & 55.42 & 38.03 & 0.10 \\
\hline SMG & 100 & 1,425 & 0.78 & 14 & 43.02 & 27.08 & 0.14 \\
\hline SNG & 100 & 1,623 & 0.68 & 16 & 43.66 & 29.10 & 0.16 \\
\hline
\end{tabular}

(0.03); on the other hand, the highest diversity of tree species (Shannon-Wiener Diversity Index) was found in KBG, Golaghat (3.78,) and it was the lowest in DG, Jorhat (2.52). However, species evenness index of tree species ranged from 0.77 to 0.93 with a mean of $0.86 \pm 0.01$ and it was peak in KBG, Golaghat, and the lowest in KNG, Golaghat. Sørensen's similarity indices showed a high degree of similarity with a range from $34.25 \%$ to $75.38 \%$ among different study sites (Table 4).

\section{Discussion}

Floristic inventory, survey, and diversity studies help us to understand the species composition and diversity status of any community [19]. A. malaccensis is one of the most dominant tree species in study sites of upper Assam representing $10-54 \%$ of the total tree density (with a mean of $34 \% \pm 2$ ) showing a trend towards monoculture. In general, monoculture has a great impact on species diversity. Introduction of rubber (Hevea brasiliensis) into homegardens resulted in a reduction of species diversity in homegardens of Kerela [20]. But this is not evident in the present study and may be due to the fact that owners tried to produce all the possible species of common household utilities in their homegarden itself.

Density of a species provides an index to competition between individuals of the species. Higher densities can cause greater competitive stresses, leading to poor growth and lower reproductive capacity. We recorded $A$. malaccensis with high density (429 to 6,236 individuals ha ${ }^{-1}$ with a mean of 1,609 individuals $\mathrm{ha}^{-1} \pm 217$ ), frequency ( $93 \%$ to $100 \%$ with a mean of $98 \% \pm 0.53$ ), and abundance ( 4 to 62 with a mean of $16 \pm 2$ ). One potential explanation of higher density, frequency, and abundance could be extensive cultivation of the species in study sites because of its high commercial value. Total basal cover of trees $(>3.18 \mathrm{~cm} \mathrm{DBH})$ is very low and ranged from $0.34 \mathrm{~cm}^{2} \mathrm{ha}^{-1}$ to $1.25 \mathrm{~cm}^{2} \mathrm{ha}^{-1}$ with a mean of $0.67 \mathrm{~cm}^{2} \mathrm{ha}^{-1} \pm 0.04$, may be because of the highest density of lower diameter class individuals of $A$. malaccensis. Importance value index (IVI) of the species represents the ecological success of any species in a community and it gives an excellent idea about varying environmental factors [21]. It provides a complete picture of sociological structure of a species, because the frequency provides information about the dispersal of a species in an area, density gives numerical strength of the species, and dominance represents the basal area. In almost all study sites, the highest IVI is contributed 


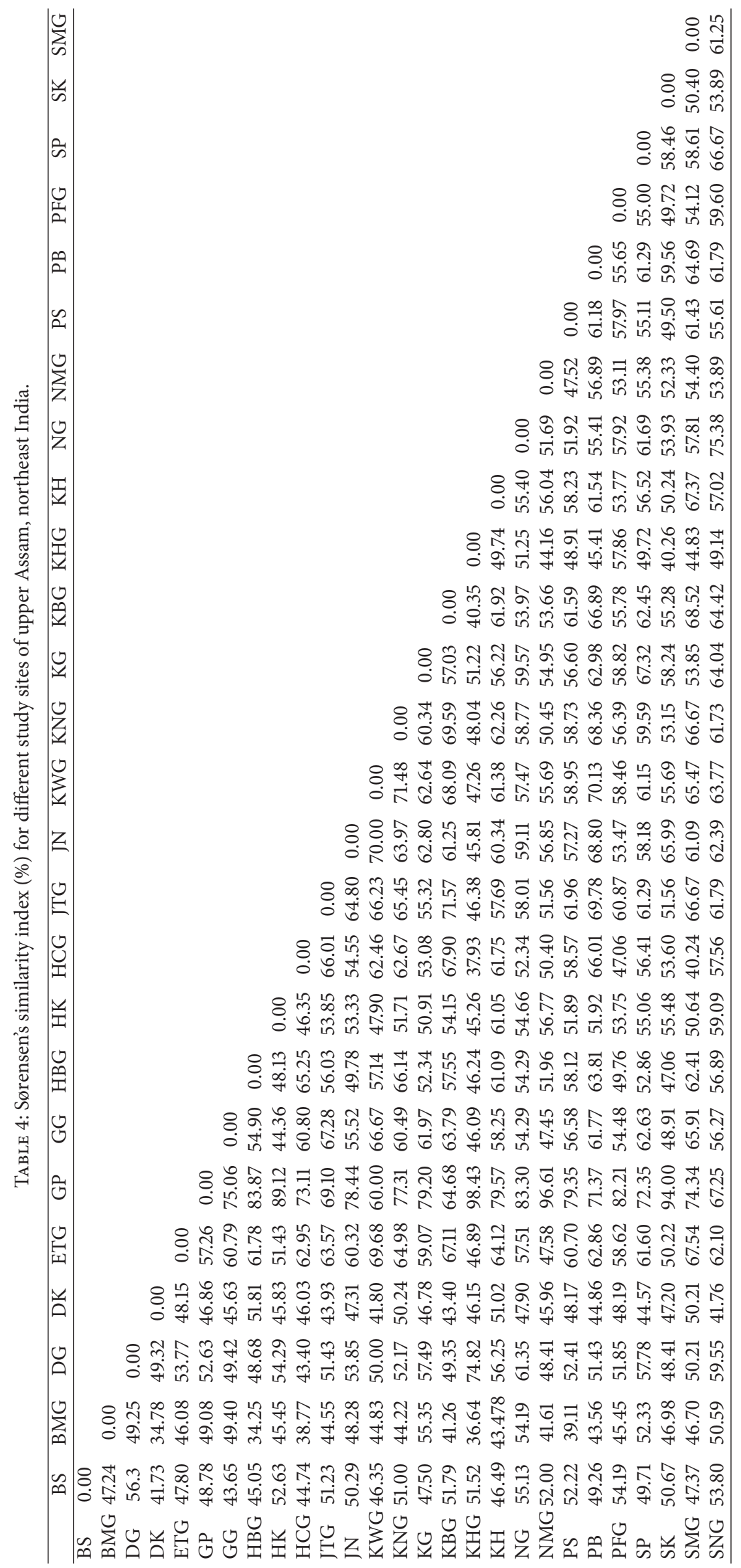


TABle 5: Density (individuals $\left.\mathrm{ha}^{-1}\right)$, frequency (\%), abundance, and IVI of tree $(>3.18 \mathrm{~cm} \mathrm{DBH})$, shrub, and herb species in studied homegardens of upper Assam, northeast India.

\begin{tabular}{|c|c|c|c|c|c|}
\hline Species & Family & Density (individuals ha ${ }^{-1}$ ) & Frequency (\%) & Abundance & IVI \\
\hline \multicolumn{6}{|c|}{ Trees } \\
\hline Acacia auriculiformis Cunn. ex Benth. & Mimosaceae & 1.06 & 0.66 & 2 & 1.76 \\
\hline Adenanthera pavonina $\mathrm{L}$. & Mimosaceae & 2.38 & 2.11 & 1 & 0.94 \\
\hline Aegle marmelos Corr. & Rutaceae & 2.64 & 2.51 & 1 & 1.00 \\
\hline Ailanthus grandis Prain & Simaroubaceae & 0.13 & 0.13 & 1 & 0.87 \\
\hline Albizia lebbeck (L.) Benth. & Mimosaceae & 2.11 & 1.32 & 2 & 2.30 \\
\hline Albizia lucida (Roxb.) Benth. & Mimosaceae & 7.53 & 5.28 & 1 & 1.68 \\
\hline Albizia procera (Roxb.) Benth. & Mimosaceae & 0.66 & 0.66 & 1 & 0.68 \\
\hline Alstonia scholaris (L.) R. Br. & Apocynaceae & 15.85 & 11.89 & 1 & 2.90 \\
\hline Amoora wallichii King & Meliaceae & 0.66 & 0.53 & 1 & 0.42 \\
\hline Anacardium occidentale L. & Anacardiaceae & 0.26 & 0.26 & 1 & 0.71 \\
\hline Annona reticulata $\mathrm{L}$. & Annonaceae & 1.06 & 1.06 & 1 & 0.39 \\
\hline $\begin{array}{l}\text { Anthocephalus chinensis (Lam.) Rich. ex } \\
\text { Walp. }\end{array}$ & Rubiaceae & 5.81 & 4.49 & 1 & 2.22 \\
\hline Antidesma ghaesembilla Gaert. & Euphorbiaceae & 0.40 & 0.40 & 1 & 0.21 \\
\hline Aquilaria malaccensis Lam. & Thymelaeaceae & 1442.67 & 98.02 & 15 & 49.50 \\
\hline Arabongali* $^{*}$ & & 0.26 & 0.26 & 1 & 0.21 \\
\hline Araucaria heterophylla (Salisb.) Franco & Araucariaceae & 0.53 & 0.53 & 1 & 0.32 \\
\hline Areca catechu L. & Arecaceae & 554.29 & 74.37 & 7 & 24.94 \\
\hline Artocarpus chaplasha Roxb. & Moraceae & 2.25 & 1.59 & 1 & 1.46 \\
\hline Artocarpus heterophyllus Lam. & Moraceae & 34.87 & 24.57 & 1 & 5.49 \\
\hline Artocarpus lakoocha Roxb. & Moraceae & 3.04 & 2.77 & 1 & 1.83 \\
\hline Averrhoa carambola $\mathrm{L}$. & Averrhoaceae & 4.49 & 4.23 & 1 & 1.19 \\
\hline Azadirachta indica Juss. & Meliaceae & 1.19 & 1.19 & 1 & 0.43 \\
\hline Baccaurea sapida (Roxb.) Mull. Arg. & Euphorbiaceae & 1.32 & 1.19 & 1 & 0.36 \\
\hline Bambusa balcooa Roxb. & Poaceae & 214.93 & 3.04 & 71 & 5.53 \\
\hline Bambusa nutans G. C. Wall. ex Munro & Poaceae & 2.25 & 0.13 & 17 & 0.07 \\
\hline Bambusa pallida Munro. & Poaceae & 1157.60 & 11.23 & 103 & 28.96 \\
\hline Bambusa tulda Roxb. & Poaceae & 50.99 & 0.53 & 97 & 1.28 \\
\hline Bauhinia purpurea L. & Caesalpiniaceae & 0.26 & 0.26 & 1 & 0.46 \\
\hline Beilschmiedia brandisii Hook. $\mathrm{f}$. & Lauraceae & 1.45 & 0.79 & 2 & 0.48 \\
\hline Bixa orellana L. & Bixaceae & 2.38 & 1.72 & 1 & 0.54 \\
\hline Bombax ceiba $\mathrm{L}$. & Malvaceae & 3.83 & 3.43 & 1 & 1.75 \\
\hline Borassus flabellifer L. & Arecaceae & 0.13 & 0.13 & 1 & 1.74 \\
\hline Bridelia retusa (L.) A. Juss. & Euphorbiaceae & 0.66 & 0.40 & 2 & 0.66 \\
\hline Butea monosperma (Lam.) Taubert & Papilionaceae & 0.40 & 0.26 & 2 & 0.95 \\
\hline Callistemon lanceolatus D.C. & Myrtaceae & 0.53 & 0.40 & 1 & 0.29 \\
\hline Canarium strictum Roxb. & Burseraceae & 0.53 & 0.53 & 1 & 1.07 \\
\hline Carallia lucida Roxb. ex Kurz. & Rhizophoraceae & 1.72 & 1.59 & 1 & 0.52 \\
\hline Carica papaya L. & Caricaceae & 34.48 & 18.63 & 2 & 3.95 \\
\hline Caryota urens L. & Arecaceae & 1.98 & 1.59 & 1 & 1.13 \\
\hline Cassia fistula $\mathrm{L}$. & Caesalpiniaceae & 12.15 & 7.79 & 2 & 2.04 \\
\hline Cassia siamea Lam. & Caesalpiniaceae & 1.85 & 1.72 & 1 & 0.86 \\
\hline Castanopsis indica (Roxb. ex Lindl.) A. D.C. & Fagaceae & 1.19 & 0.66 & 2 & 0.44 \\
\hline Chukrasia tabularis Juss. & Meliaceae & 1.06 & 0.79 & 1 & 0.92 \\
\hline Cinnamomum glanduliferum (Wall.) Meisn. & Lauraceae & 0.26 & 0.26 & 1 & 1.10 \\
\hline $\begin{array}{l}\text { Cinnamomum tamala (Buch.-Ham.) Nees } \\
\text { and Eberm. }\end{array}$ & Lauraceae & 5.28 & 4.36 & 1 & 1.05 \\
\hline
\end{tabular}


TABle 5: Continued.

\begin{tabular}{|c|c|c|c|c|c|}
\hline Species & Family & Density (individuals ha ${ }^{-1}$ ) & Frequency (\%) & Abundance & IVI \\
\hline Citrus limon (L.) Burm.f. & Rutaceae & 7.79 & 4.76 & 2 & 0.97 \\
\hline Citrus maxima (Merr.) & Rutaceae & 10.44 & 9.25 & 1 & 1.91 \\
\hline Citrus medica L. & Rutaceae & 0.92 & 0.53 & 2 & 0.13 \\
\hline Citrus reticulata Blanco. & Rutaceae & 7.13 & 4.76 & 2 & 0.97 \\
\hline Citrus sinensis (L.) Osbeck & Rutaceae & 1.06 & 0.66 & 2 & 0.18 \\
\hline Citrus sp. & Rutaceae & 2.38 & 1.85 & 1 & 0.38 \\
\hline Cocos nucifera L. & Arecaceae & 25.76 & 17.83 & 1 & 4.38 \\
\hline Cordia grandis Roxb. & Boraginaceae & 2.64 & 2.11 & 1 & 0.82 \\
\hline Crataeva nurvala Ham. & Capparidaceae & 1.32 & 1.19 & 1 & 0.53 \\
\hline Dalhousiea bracteata (Roxb.) Baker & Papilionaceae & 0.13 & 0.13 & 1 & 0.30 \\
\hline Delonix regia (Boj. ex Hook.) Raf. & Caesalpinaceae & 2.38 & 1.85 & 1 & 0.87 \\
\hline Dillenia indica L. & Dilleniaceae & 0.66 & 0.66 & 1 & 3.00 \\
\hline Diospyros embryopteris Pers. & Ebenaceae & 0.53 & 0.40 & 1 & 1.24 \\
\hline $\begin{array}{l}\text { Dysoxylum binectariferum (Roxb.) Hook. f. } \\
\text { ex Bedd. }\end{array}$ & Meliaceae & 2.64 & 1.98 & 1 & 1.08 \\
\hline Ehretia acuminata R.Br. & Boraginaceae & 1.06 & 1.06 & 1 & 0.71 \\
\hline Elaeocarpus floribundus Blume. & Elaeocarpaceae & 5.55 & 5.02 & 1 & 1.56 \\
\hline Elaeocarpus ganitrus Roxb. & Elaeocarpaceae & 0.13 & 0.13 & 1 & 1.29 \\
\hline Erythrina stricta Roxb. & Papilionaceae & 2.51 & 1.59 & 2 & 0.67 \\
\hline Eucalyptus citriodora Hook. & Myrtaceae & 0.79 & 0.79 & 1 & 0.95 \\
\hline Eugenia fruticosa Roxb. & Myrtaceae & 1.59 & 1.32 & 1 & 0.57 \\
\hline Eugenia jambolana Lam. & Myrtaceae & 5.55 & 4.49 & 1 & 1.39 \\
\hline $\begin{array}{l}\text { Eugenia jambolana Lam. var. caryophyllifolia } \\
\text { Duthie. }\end{array}$ & Myrtaceae & 0.40 & 0.40 & 1 & 0.63 \\
\hline Eugenia jambos L. & Myrtaceae & 3.04 & 2.91 & 1 & 0.73 \\
\hline Evodia meliaefolia Benth. & Rutaceae & 2.77 & 2.38 & 1 & 1.13 \\
\hline Ficus benghalensis $\mathrm{L}$. & Moraceae & 0.26 & 0.26 & 1 & 9.66 \\
\hline Ficus cyrtophylla & Moraceae & 0.53 & 0.13 & 1 & 0.03 \\
\hline Ficus fistulosa Reinw. ex Blume & Moraceae & 0.53 & 0.53 & 2 & 9.66 \\
\hline Ficus hirta Vahl. & Moraceae & 1.06 & 0.13 & 1 & 0.04 \\
\hline Ficus hispida L. & Moraceae & 5.28 & 3.96 & 25 & 0.89 \\
\hline Ficus racemosa $\mathrm{L}$. & Moraceae & 0.40 & 0.40 & 3 & 0.39 \\
\hline Ficus religiosa $\mathrm{L}$. & Moraceae & 0.53 & 0.53 & 4 & 6.56 \\
\hline Garcinia lanceaefolia Roxb. & Clusiaceae & 0.26 & 0.26 & 1 & 0.43 \\
\hline Garcinia morella (Gaertn.) Desr. & Clusiaceae & 1.45 & 1.06 & 7 & 0.44 \\
\hline Garcinia paniculata Roxb. & Clusiaceae & 0.26 & 0.26 & 2 & 0.2 \\
\hline Garcinia pedunculata Roxb. Engl. & Clusiaceae & 0.79 & 0.79 & 6 & 0.96 \\
\hline $\begin{array}{l}\text { Garcinia xanthochymus Hook. f. ex } \\
\text { Anderson Engl. }\end{array}$ & Clusiaceae & 0.40 & 0.40 & 3 & 0.39 \\
\hline Gmelina arborea Roxb. & Verbenaceae & 10.30 & 6.87 & 36 & 2.02 \\
\hline Grevillea robusta Cunn. ex R.Br. & Proteaceae & 1.72 & 1.32 & 8 & 1.37 \\
\hline Heteropanax fragrans (Roxb.) Seem. & Araliaceae & 4.76 & 3.04 & 11 & 1.16 \\
\hline Ilex umbellulata (Wall.) Loes. & Aquifoliaceae & 1.32 & 1.19 & 8 & 0.38 \\
\hline Lagerstroemia flos reginae Retz. & Lythraceae & 6.08 & 4.89 & 24 & 1.53 \\
\hline Litchi chinensis Sonn. & Sapindaceae & 6.34 & 5.55 & 36 & 1.43 \\
\hline Litsea elongata Wall. & Lauraceae & 0.79 & 0.53 & 3 & 0.32 \\
\hline Litsea monopetala (Roxb.) Pers. & Lauraceae & 10.30 & 8.19 & 45 & 2.01 \\
\hline Litsea nitida Hook. f. & Lauraceae & 12.29 & 8.06 & 37 & 1.86 \\
\hline
\end{tabular}


TABLE 5: Continued.

\begin{tabular}{|c|c|c|c|c|c|}
\hline Species & Family & Density (individuals ha ${ }^{-1}$ ) & Frequency (\%) & Abundance & IVI \\
\hline Litsea sebifera Pers. & Lauraceae & 1.19 & 1.19 & 7 & 0.47 \\
\hline Livistona jenkinsiana Griff. & Arecaceae & 1.72 & 1.45 & 7 & 1.2 \\
\hline Macaranga peltata (Roxb.) Mueller & Euphorbiaceae & 0.13 & 0.13 & 1 & 0.22 \\
\hline Magnolia sphenocarpa Roxb. & Magnoliaceae & 4.76 & 3.17 & 16 & 0.99 \\
\hline Mallotus philippinensis Muell. Arg. & Euphorbiaceae & 3.57 & 3.43 & 24 & 0.84 \\
\hline Mangifera indica $\mathrm{L}$. & Anacardiaceae & 62.48 & 35.80 & 115 & 7.79 \\
\hline Manilkara zapota (L.) van Royen & Sapotaceae & 0.13 & 0.13 & 1 & 1.07 \\
\hline Melia azedarach L. & Meliaceae & 9.38 & 7.27 & 37 & 1.61 \\
\hline Mesua ferrea L. & Clusiaceae & 5.15 & 3.57 & 24 & 1.01 \\
\hline Michelia champaca L. & Magnoliaceae & 2.25 & 1.19 & 6 & 0.75 \\
\hline Mimusops elengi L. & Sapotaceae & 0.66 & 0.66 & 5 & 1.42 \\
\hline Moringa oleifera Lam. & Moringaceae & 4.62 & 4.10 & 29 & 1.15 \\
\hline Morus alba L. & Moraceae & 2.25 & 1.32 & 9 & 0.56 \\
\hline Morus laevigata Wall. & Moraceae & 0.13 & 0.13 & 1 & 0.2 \\
\hline Murraya koenigii (L.) Sprengel. & Rutaceae & 2.38 & 1.85 & 12 & 0.49 \\
\hline Murraya paniculata (L.) Jack & Rutaceae & 0.13 & 0.13 & 1 & 0.19 \\
\hline Musa acuminata Colla. & Musaceae & 64.33 & 12.42 & 57 & 3.64 \\
\hline Musa balbisiana Colla. & Musaceae & 137.38 & 24.83 & 92 & 7.4 \\
\hline Musa calosperma F. Muell. & Musaceae & 72.26 & 14.40 & 62 & 4.14 \\
\hline Musa paradisiaca L. & Musaceae & 0.53 & 0.26 & 2 & 0.19 \\
\hline Musa sp. & Musaceae & 12.42 & 3.04 & 17 & 0.98 \\
\hline Myrica esculenta Buch.-Ham. ex D. Don & Myricaceae & 2.64 & 1.98 & 12 & 0.7 \\
\hline Oroxylum indicum (L.) Kurz. & Bignoniaceae & 5.02 & 4.36 & 24 & 1.13 \\
\hline Pachylarnax pleiocarpa Dandy & Magnoliaceae & 0.92 & 0.79 & 5 & 0.96 \\
\hline Persea bombycina (King ex Hook. f.) Kost. & Lauraceae & 3.04 & 2.25 & 15 & 0.86 \\
\hline Phoebe goalparensis Hutch. & Lauraceae & 0.13 & 0.13 & 1 & 0.19 \\
\hline Phoenix dactylifera L. & Arecaceae & 0.53 & 0.40 & 2 & 1.04 \\
\hline Phyllanthus acidus (L.) Skeels. & Phyllanthaceae & 1.06 & 1.06 & 8 & 0.35 \\
\hline Phyllanthus emblica L. & Phyllanthaceae & 5.15 & 5.02 & 31 & 1.26 \\
\hline Plumeria rubra L. & Apocynaceae & 0.26 & 0.13 & 1 & 0.24 \\
\hline Polyalthia longifolia (Sonn.) Thw. & Annonaceae & 6.87 & 2.64 & 14 & 0.76 \\
\hline Pongamia pinnata (L.) Pierre. & Papilionaceae & 1.72 & 1.45 & 10 & 0.71 \\
\hline Premna bengalensis $\mathrm{Cl}$. & Verbenaceae & 5.55 & 4.49 & 25 & 1.14 \\
\hline Prunus cerasifera Ehrh. & Rosaceae & 1.59 & 1.32 & 8 & 0.46 \\
\hline Prunus jenkinsii Hook. f. and Thoms. & Rosaceae & 0.53 & 0.53 & 3 & 0.21 \\
\hline Prunus persica (L.) Batsch. & Rosaceae & 0.92 & 0.92 & 6 & 0.31 \\
\hline Psidium gujava L. & Myrtaceae & 16.64 & 12.42 & 63 & 2.5 \\
\hline Pyrus communis L. & Rosaceae & 1.06 & 0.79 & 4 & 0.26 \\
\hline Salix tetrasperma Roxb. & Salicaceae & 4.49 & 2.11 & 10 & 0.84 \\
\hline Samanea saman (Jacq.) Merr. & Mimosaceae & 0.26 & 0.26 & 2 & 4.03 \\
\hline Sapindus mukorossi Gaertn. & Sapindaceae & 1.85 & 1.72 & 10 & 0.64 \\
\hline Sapium baccatum Roxb. & Euphorbiaceae & 1.32 & 1.06 & 4 & 0.63 \\
\hline Sapium eugeniaefolium Ham. & Euphorbiaceae & 0.53 & 0.53 & 4 & 0.53 \\
\hline Schimawallichii (DC.) Korth. & Theaceae & 4.76 & 2.91 & 16 & 1.12 \\
\hline Shorea robusta Gaertn. f. & Dipterocarpaceae & 0.13 & 0.13 & 1 & 2.92 \\
\hline Spondias mangifera Wild. & Anacardiaceae & 1.19 & 0.92 & 7 & 0.54 \\
\hline Stereospermum chelonoides DC. & Sterculiaceae & 0.92 & 0.66 & 5 & 0.38 \\
\hline
\end{tabular}


TABle 5: Continued.

\begin{tabular}{|c|c|c|c|c|c|}
\hline Species & Family & Density (individuals ha ${ }^{-1}$ ) & Frequency (\%) & Abundance & IVI \\
\hline Streblus asper Lour. & Moraceae & 3.70 & 2.11 & 12 & 0.73 \\
\hline Symplocos grandiflora Wall. & Symplocaceae & 0.13 & 0.13 & 1 & 0.22 \\
\hline Talauma hodgsonii Hook. f. and Thomson & Magnoliaceae & 0.13 & 0.13 & 1 & 4.65 \\
\hline Talauma rabaniana Hk.ef. and Th. & Magnoliaceae & 0.40 & 0.40 & 2 & 2.06 \\
\hline Tamarindus indica $\mathrm{L}$. & Caesalpiniaceae & 0.26 & 0.26 & 2 & 0.43 \\
\hline Tectona grandis L. & Verbenaceae & 25.36 & 9.78 & 40 & 2.83 \\
\hline $\begin{array}{l}\text { Terminalia arjuna (Roxb. ex DC.) Wight and } \\
\text { Arn. }\end{array}$ & Combretaceae & 0.26 & 0.26 & 2 & 0.57 \\
\hline Terminalia bellirica (Gaertn.) Roxb. & Combretaceae & 0.13 & 0.13 & 1 & 0.16 \\
\hline Terminalia catappa L. & Combretaceae & 0.40 & 0.40 & 3 & 0.89 \\
\hline Terminalia chebula Retz. & Combretaceae & 14.66 & 12.42 & 64 & 2.95 \\
\hline $\begin{array}{l}\text { Terminalia myriocarpa Van Heurck and } \\
\text { Muell. Arg. }\end{array}$ & Combretaceae & 0.26 & 0.26 & 2 & 0.39 \\
\hline Toona ciliata Roem. & Meliaceae & 13.87 & 10.30 & 45 & 2.88 \\
\hline Tricalysia singularis (Korth.) K. Schum. & Rubiaceae & 0.66 & 0.40 & 3 & 0.66 \\
\hline Vernonia travancorica J. Hk. & Asteraceae & 0.13 & 0.13 & 1 & 0.04 \\
\hline Vitex negundo L. & Verbenaceae & 0.79 & 0.79 & 6 & 0.4 \\
\hline Walsura robusta Roxb. & Meliaceae & 0.40 & 0.40 & 3 & 0.38 \\
\hline Zanthoxylum rhetsa (Roxb.) DC. & Rutaceae & 1.19 & 1.06 & 8 & 0.71 \\
\hline Ziziphus mauritiana Lam. & Rhamnaceae & 4.62 & 4.36 & 27 & 1.26 \\
\hline Ziziphus sp. & Rhamnaceae & 0.13 & 0.13 & 1 & 0.42 \\
\hline \multicolumn{6}{|c|}{ Shrubs } \\
\hline Acacia farnesiana (L.) Willd. & Mimosaceae & 7.40 & 1.72 & 1.08 & 0.41 \\
\hline Adhatoda vasica Nees & Acanthaceae & 79.26 & 4.10 & 4.84 & 1.52 \\
\hline Bisalyakarani* $^{*}$ & & 5.28 & 0.13 & 10.00 & 0.07 \\
\hline Bougainvillea spectabilis Wild. & Nyctaginaceae & 5.81 & 1.32 & 1.10 & 0.32 \\
\hline Caesalpinia pulcherrima L. & Caesalpiniaceae & 2.11 & 0.53 & 1.00 & 0.13 \\
\hline Cajanus cajan (L.) Millsp. & Papilionaceae & 28.01 & 1.72 & 4.08 & 0.59 \\
\hline Calamus tenuis Roxb. & Arecaceae & 43.33 & 2.38 & 4.56 & 0.86 \\
\hline Callicarpa arborea Roxb. & Verbenaceae & 11.10 & 0.79 & 3.50 & 0.26 \\
\hline Calotropis procera (Ait.) Ait. f. & Asclepiadaceae & 4.76 & 0.92 & 1.29 & 0.23 \\
\hline Camellia sinensis (L.) O. Kuntze & Theaceae & 1958.78 & 21.14 & 23.17 & 21.23 \\
\hline Capsicum annum L. & Solanaceae & 67.11 & 5.42 & 3.10 & 1.68 \\
\hline Capsicum annum L. var. longum & Solanaceae & 86.13 & 6.74 & 3.20 & 2.12 \\
\hline Capsicum chinense Jacq. & Solanaceae & 61.29 & 6.21 & 2.47 & 1.80 \\
\hline Cassia alata L. & Caesalpinaceae & 11.10 & 0.92 & 3.00 & 0.28 \\
\hline Cassia sophera L. & Caesalpinaceae & 5.28 & 0.79 & 1.67 & 0.21 \\
\hline Cassia tora $\mathrm{L}$. & Caesalpinaceae & 7.93 & 0.53 & 3.75 & 0.18 \\
\hline $\begin{array}{l}\text { Chromolaena odorata (L.) King and H.E. } \\
\text { Robins. }\end{array}$ & Asteraceae & 528.40 & 18.23 & 7.25 & 8.28 \\
\hline Clerodendrum colebrookianum Wall. & Verbenaceae & 33.82 & 2.51 & 3.37 & 0.80 \\
\hline Clerodendrum japonicum (Thunb.) Sweet & Verbenaceae & 22.72 & 1.85 & 3.07 & 0.57 \\
\hline Clerodendrum viscosum Vent. & Verbenaceae & 984.94 & 29.59 & 8.32 & 14.54 \\
\hline Codiaeum variegatum (L.) Juss. & Euphorbiaceae & 56.01 & 7.13 & 1.96 & 1.94 \\
\hline Coffea benghalensis Roxb. ex Schult. & Rubiaceae & 18.49 & 1.59 & 2.92 & 0.48 \\
\hline Dracaena steudneri Engl. & Dracaenaceae & 5.28 & 0.79 & 1.67 & 0.21 \\
\hline Euonymus japonicus Thunb. & Celastraceae & 144.25 & 6.87 & 5.25 & 2.65 \\
\hline Euphorbia neriifolia L. & Euphorbiaceae & 8.45 & 1.72 & 1.23 & 0.42 \\
\hline Flacourtia cataphracta Roxb. ex Willd. & Flacourtiaceae & 11.10 & 2.38 & 1.17 & 0.58 \\
\hline
\end{tabular}


TABle 5: Continued.

\begin{tabular}{|c|c|c|c|c|c|}
\hline Species & Family & Density (individuals ha $^{-1}$ ) & Frequency (\%) & Abundance & IVI \\
\hline Flemingia strobilifera (L.) R. Br. & Papilionaceae & 22.19 & 2.38 & 2.33 & 0.68 \\
\hline Gardenia jasminoides Ellis. & Rubiaceae & 41.22 & 5.94 & 1.73 & 1.57 \\
\hline Glochidion arborescens $\mathrm{Bl}$. & Euphorbiaceae & 1.06 & 0.26 & 1.00 & 0.06 \\
\hline Gossypium arboreum L. & Malvaceae & 25.36 & 4.62 & 1.37 & 1.16 \\
\hline Hibiscus mutabilis L. & Malvaceae & 4.23 & 0.92 & 1.14 & 0.23 \\
\hline Hibiscus rosa-sinensis $\mathrm{L}$. & Malvaceae & 76.09 & 9.25 & 2.06 & 2.54 \\
\hline Hibiscus sabdariffa L. & Malvaceae & 57.07 & 3.30 & 4.32 & 1.17 \\
\hline Ixora javanica (Blume) DC. & Rubiaceae & 22.72 & 2.64 & 2.15 & 0.74 \\
\hline Jasminum officinale L. & Oleaceae & 6.87 & 1.45 & 1.18 & 0.36 \\
\hline Jatropha gossypiifolia L. & Euphorbiaceae & 5.28 & 0.79 & 1.67 & 0.21 \\
\hline Justicia gendarussa Blanco. & Acanthaceae & 56.54 & 2.11 & 6.69 & 0.92 \\
\hline Lawsonia inermis $\mathrm{L}$. & Lawsoniaceae & 10.57 & 2.25 & 1.18 & 0.55 \\
\hline Leea indica (Burm. f.) Merr. & Leeaceae & 11.62 & 1.45 & 2.00 & 0.40 \\
\hline $\begin{array}{l}\text { Malvaviscus arboreus Cav. var. drummondii } \\
\text { (Torr. and Gray) Schery. }\end{array}$ & Malvaceae & 14.27 & 1.98 & 1.80 & 0.53 \\
\hline Melastoma normale D. Don. & Melastomataceae & 70.81 & 4.49 & 3.94 & 1.53 \\
\hline Mussaenda frondosa $\mathrm{L}$. & Rubiaceae & 28.01 & 3.57 & 1.96 & 0.97 \\
\hline Nerium indicum Mill. & Apocynaceae & 11.10 & 2.11 & 1.31 & 0.53 \\
\hline Nyctanthes arbor-tristis L. & Oleaceae & 26.95 & 5.68 & 1.19 & 1.39 \\
\hline Ocimum gratissimum $\mathrm{L}$. & Lamiaceae & 8.45 & 1.06 & 2.00 & 0.29 \\
\hline Ocimum sanctum $L$. & Lamiaceae & 67.64 & 6.21 & 2.72 & 1.85 \\
\hline Phlogacanthus thyrsiflorus (Roxb.) Nees & Acanthaceae & 38.04 & 2.11 & 4.50 & 0.76 \\
\hline $\begin{array}{l}\text { Pogostemon benghalensis (Burm. f.) O. } \\
\text { Kuntze }\end{array}$ & Lamiaceae & 20.61 & 0.66 & 7.80 & 0.31 \\
\hline Punica granatum $\mathrm{L}$. & Punicaceae & 12.15 & 2.64 & 1.15 & 0.64 \\
\hline Ravenala madagascariensis Sonnerat. & Strelitziaceae & 6.87 & 0.40 & 4.33 & 0.14 \\
\hline Ricinus communis L. & Euphorbiaceae & 172.79 & 5.55 & 7.79 & 2.62 \\
\hline Rosa alba $\mathrm{L}$. & Rosaceae & 33.29 & 3.17 & 2.63 & 0.93 \\
\hline Saccharum officinarum $\mathrm{L}$. & Poaceae & 239.37 & 3.70 & 16.18 & 2.82 \\
\hline Sida cordifolia L. & Malvaceae & 15.85 & 0.53 & 7.50 & 0.24 \\
\hline Solanum indicum L. & Solanaceae & 5.28 & 0.79 & 1.67 & 0.21 \\
\hline Solanum melongena L. & Solanaceae & 93.00 & 6.08 & 3.83 & 2.04 \\
\hline Solanum subtrucatum & Solanaceae & 0.53 & 0.13 & 1.00 & 0.03 \\
\hline Solanum torvum Swartz. & Solanaceae & 11.10 & 1.59 & 1.75 & 0.42 \\
\hline Tabernaemontana divaricata G. Don. & Apocynaceae & 63.94 & 7.27 & 2.20 & 2.03 \\
\hline Thuja occidentalis L. & Cupressaceae & 3.17 & 0.66 & 1.20 & 0.16 \\
\hline Urena lobata L. & Malvaceae & 172.79 & 6.47 & 6.67 & 2.81 \\
\hline \multicolumn{6}{|c|}{ Herb } \\
\hline Abelmoschus esculentus (L.) Moench & Malvaceae & 244 & 0.40 & 6.17 & 0.11 \\
\hline Achyranthes aspera $\mathrm{L}$. & Amaranthaceae & 18032 & 26.02 & 6.93 & 7.83 \\
\hline Acorus calamus L. & Araceae & 185 & 0.13 & 14.00 & 0.06 \\
\hline Ageratum conyzoides L. & Asteraceae & 22120 & 37.52 & 5.90 & 10.48 \\
\hline Alocasia odora (Lindl.) Koch. & Araceae & 2583 & 7.46 & 3.46 & 1.71 \\
\hline Alpinia allughas $\mathrm{L}$. & Zingiberaceae & 20 & 0.07 & 3.00 & 0.01 \\
\hline Alternanthera sessilis (L.) R. Br. ex DC. & Amaranthaceae & 5713 & 10.17 & 5.62 & 2.78 \\
\hline Amaranthusblitum L. var. oleracea Hook. f. & Amaranthaceae & 535 & 0.99 & 5.40 & 0.27 \\
\hline Amaranthus caudatus L. & Amaranthaceae & 145 & 0.73 & 2.00 & 0.14 \\
\hline
\end{tabular}


TABle 5: Continued.

\begin{tabular}{|c|c|c|c|c|c|}
\hline Species & Family & Density (individuals ha ${ }^{-1}$ ) & Frequency $(\%)$ & Abundance & IVI \\
\hline Amaranthus spinosus L. & Amaranthaceae & 264 & 0.59 & 4.44 & 0.15 \\
\hline Amaranthus viridis $\mathrm{L}$. & Amaranthaceae & 7345 & 9.58 & 7.67 & 3.03 \\
\hline $\begin{array}{l}\text { Amorphophallus campanulatus (Roxb.) Bl. } \\
\text { ex Decne. }\end{array}$ & Araceae & 304 & 0.79 & 3.83 & 0.19 \\
\hline Ananas comosus (L.) Merr. & Bromeliaceae & 7999 & 9.78 & 8.18 & 3.20 \\
\hline Asparagus racemosus Willd. & Asparagaceae & 26 & 0.13 & 2.00 & 0.03 \\
\hline Atriplex hortensis L. & Chenopodiaceae & 277 & 0.46 & 6.00 & 0.13 \\
\hline Axonopus sp. & Poaceae & 60885 & 34.28 & 17.76 & 18.07 \\
\hline Bacopa monnieri (L.) Pennell & Scrophulariaceae & 383 & 0.40 & 9.67 & 0.14 \\
\hline Benincasa hispida (Thunb) Cogn. & Cucurbitaceae & 178 & 0.92 & 1.93 & 0.18 \\
\hline Borreria articularis (L. f.) F. N. Will. & Rubiaceae & 18336 & 23.91 & 7.67 & 7.57 \\
\hline Borreria hispida (L.) K. Schum. & Rubiaceae & 2272 & 4.62 & 4.91 & 1.20 \\
\hline $\begin{array}{l}\text { Brassica juncea (L.) Czern. and Cross. var. } \\
\text { cuneifolia (Roxb.) Kitam. }\end{array}$ & Brassicaceae & 2067 & 1.65 & 12.52 & 0.69 \\
\hline Brassica oleracea L. var. botrytis L. & Brassicaceae & 515 & 0.59 & 8.67 & 0.20 \\
\hline Brassica oleracea L. var. capitata L. & Brassicaceae & 608 & 0.73 & 8.36 & 0.24 \\
\hline Brassica oleracea L. var. gongylodes L. & Brassicaceae & 872 & 1.12 & 7.76 & 0.36 \\
\hline Bryophyllum pinnatum Lam. & Crassulaceae & 1849 & 4.43 & 4.18 & 1.08 \\
\hline Cannabis sativa $\mathrm{L}$. & Cannabaceae & 661 & 1.52 & 4.35 & 0.38 \\
\hline Carex sp. & Cyperaceae & 211 & 0.46 & 4.57 & 0.12 \\
\hline Catharanthus roseus $\mathrm{L}$. & Apocynaceae & 211 & 0.79 & 2.67 & 0.17 \\
\hline Celosia sp. & Amaranthaceae & 159 & 0.53 & 3.00 & 0.12 \\
\hline Centella asiatica (L.) Urban & Apiaceae & 7325 & 13.80 & 5.31 & 3.69 \\
\hline Chenopodium album $\mathrm{L}$. & Chenopodiaceae & 251 & 0.86 & 2.92 & 0.19 \\
\hline Chrysopogon aciculatus (Retz.) Trin. & Poaceae & 6532 & 3.76 & 17.35 & 1.95 \\
\hline Cleome viscosa $\mathrm{L}$. & Capparaceae & 192 & 0.79 & 2.42 & 0.16 \\
\hline Clitoria ternatea $\mathrm{L}$. & Papilionaceae & 46 & 0.40 & 1.17 & 0.07 \\
\hline Coccinia cordifolia (L.) Cogn. & Cucurbitaceae & 185 & 0.92 & 2.00 & 0.18 \\
\hline Coleus sp. & Lamiaceae & 159 & 0.33 & 4.80 & 0.08 \\
\hline Colocasia esculenta (L.) Schott & Araceae & 13349 & 20.94 & 6.38 & 6.06 \\
\hline Commelina benghalensis L. & Commelinaceae & 4505 & 8.72 & 5.17 & 2.30 \\
\hline Coriandrum sativum $\mathrm{L}$. & Apiaceae & 1572 & 0.59 & 26.44 & 0.42 \\
\hline Costus speciosus (Koen. ex Retz.) Smith & Costaceae & 159 & 0.66 & 2.40 & 0.14 \\
\hline Crassocephalum crepidioides (Benth.) Moore. & Asteraceae & 462 & 1.25 & 3.68 & 0.29 \\
\hline Cucumis sativus L. & Cucurbitaceae & 159 & 0.46 & 3.43 & 0.11 \\
\hline Cucurbita pepo L. & Cucurbitaceae & 297 & 1.32 & 2.25 & 0.27 \\
\hline Cuphea carthagenensis (Jacq.) JF Macbr. & Lythraceae & 1955 & 3.83 & 5.10 & 1.01 \\
\hline Curcuma longa L. & Zingiberaceae & 4914 & 3.43 & 14.31 & 1.56 \\
\hline Cynodon dactylon (L.) Pers. & Poaceae & 20086 & 19.29 & 10.41 & 7.21 \\
\hline Cyperus brevifolius (Rottb.) Endl. ex Hassk. & Cyperaceae & 3554 & 6.08 & 5.85 & 1.69 \\
\hline Cyperus rotundus $\mathrm{L}$. & Cyperaceae & 14650 & 20.34 & 7.20 & 6.24 \\
\hline Cyperus sp. & Cyperaceae & 6968 & 13.41 & 5.20 & 3.55 \\
\hline Daucus carota L. var. sativa DC. & Apiaceae & 1770 & 0.99 & 17.87 & 0.52 \\
\hline Desmodium elegans DC. & Papilionaceae & 26 & 0.13 & 2.00 & 0.03 \\
\hline Dioscorea alata L. & Dioscoreaceae & 476 & 2.11 & 2.25 & 0.43 \\
\hline Dioscorea deltoidea Wall. & Dioscoreaceae & 40 & 0.26 & 1.50 & 0.05 \\
\hline Diplazium esculentum (Retz.) Sw. & Athyriaceae & 1830 & 4.76 & 3.85 & 1.13 \\
\hline Dolichos lablab L. & Papilionaceae & 132 & 0.79 & 1.67 & 0.15 \\
\hline
\end{tabular}


TABLE 5: Continued.

\begin{tabular}{|c|c|c|c|c|c|}
\hline Species & Family & Density (individuals ha ${ }^{-1}$ ) & Frequency (\%) & Abundance & IVI \\
\hline $\begin{array}{l}\text { Drymaria cordata (L.) Wild ex Roemer and } \\
\text { Schultes }\end{array}$ & Caryophyllaceae & 7919 & 9.51 & 8.33 & 3.14 \\
\hline Dryopteris sp. & Dryopteridaceae & 12450 & 18.23 & 6.83 & 5.45 \\
\hline Eclipta prostrata L. & Asteraceae & 139 & 0.86 & 1.62 & 0.16 \\
\hline Eryngium foetidum L. & Apiaceae & 489 & 1.06 & 4.63 & 0.27 \\
\hline Houttuynia cordata Thunb. & Saururaceae & 1836 & 1.65 & 11.12 & 0.64 \\
\hline Impatiens balsamina $\mathrm{L}$. & Balsaminaceae & 1440 & 2.51 & 5.74 & 0.69 \\
\hline Imperata cylindrica (L.) Raeusch. & Poaceae & 264 & 0.07 & 40.00 & 0.07 \\
\hline Justicia simplex D. Don. & Acanthaceae & 8303 & 16.91 & 4.91 & 4.38 \\
\hline Lagenaria siceraria (Mol.) Standl. & Cucurbitaceae & 244 & 1.25 & 1.95 & 0.25 \\
\hline Leucas aspera (Willd.) Spreng. & Lamiaceae & 2715 & 6.74 & 4.03 & 1.62 \\
\hline Longbon* & Rubiaceae & 1030 & 2.44 & 4.22 & 0.60 \\
\hline Luffaacutangula (L.) Roxb. & Cucurbitaceae & 462 & 1.45 & 3.18 & 0.32 \\
\hline Luffa cylindrica (L.) Roem. & Cucurbitaceae & 304 & 1.32 & 2.30 & 0.27 \\
\hline Lycopersicon esculentum Mill. & Solanaceae & 601 & 1.65 & 3.64 & 0.38 \\
\hline Mentha viridis L. & Lamiaceae & 416 & 0.53 & 7.88 & 0.17 \\
\hline Mikania micrantha Kunth ex H.B.K. & Asteraceae & 6559 & 15.92 & 4.12 & 3.86 \\
\hline Momordica charantia L. & Cucurbitaceae & 79 & 0.33 & 2.40 & 0.07 \\
\hline Momordica dioica Roxb. ex Willd & Cucurbitaceae & 410 & 1.45 & 2.82 & 0.31 \\
\hline Mimosa pudica L. & Mimosaceae & 244 & 0.99 & 2.47 & 0.21 \\
\hline Mirabilis jalapa L. & Nyctaginaceae & 462 & 1.06 & 4.38 & 0.26 \\
\hline Oldenlandia corymbosa L. & Rubiaceae & 4287 & 5.75 & 7.46 & 1.79 \\
\hline Oplismenus sp. & Poaceae & 37457 & 28.93 & 12.95 & 12.34 \\
\hline Oryza sativa $\mathrm{L}$. & Poaceae & 449 & 0.26 & 17.00 & 0.14 \\
\hline Oxalis corniculata $\mathrm{L}$. & Oxalidaceae & 4822 & 7.79 & 6.19 & 2.22 \\
\hline $\begin{array}{l}\text { Oxalis debilis H.B.K. var. corymbosa (DC.) } \\
\text { Lour. }\end{array}$ & Oxalidaceae & 5588 & 8.39 & 6.66 & 2.48 \\
\hline Paederia foetida $\mathrm{L}$. & Rubiaceae & 198 & 0.46 & 4.29 & 0.11 \\
\hline Panicum sp. & Poaceae & 38573 & 38.64 & 9.98 & 14.09 \\
\hline Passiflora foetida L. & Passifloraceae & 119 & 0.66 & 1.80 & 0.13 \\
\hline Peperomia pellucida L. & Piperaceae & 5033 & 7.79 & 6.46 & 2.27 \\
\hline Phyllanthus fraternus Webster & Euphorbiaceae & 6420 & 14.73 & 4.36 & 3.64 \\
\hline Piper betle L. & Piperaceae & 4868 & 12.62 & 3.86 & 2.99 \\
\hline Piper nigrum L. & Piperaceae & 1856 & 5.35 & 3.47 & 1.22 \\
\hline Piper sylvaticum Roxb. & Piperaceae & 627 & 0.92 & 6.79 & 0.28 \\
\hline Pollia sp. & Commelinaceae & 165 & 0.33 & 5.00 & 0.09 \\
\hline Polygonum chinense L. & Polygonaceae & 205 & 0.20 & 10.33 & 0.07 \\
\hline Polygonum hydropiper L. & Polygonaceae & 1777 & 1.85 & 9.61 & 0.66 \\
\hline Polygonum microcephalum D. Don & Polygonaceae & 159 & 0.13 & 12.00 & 0.05 \\
\hline Potentilla indica (Andrews) Wolf. & Rosaceae & 403 & 0.79 & 5.08 & 0.21 \\
\hline Pteris sp. & Pteridaceae & 1110 & 2.84 & 3.91 & 0.68 \\
\hline Raphanus sativus L. & Brassicaceae & 1004 & 0.86 & 11.69 & 0.34 \\
\hline Rhynchostylis retusa (L.) Blume. & Orchidaceae & 99 & 0.59 & 1.67 & 0.11 \\
\hline Scoparia dulcis L. & Scrophulariaceae & 1982 & 4.43 & 4.48 & 1.11 \\
\hline Selaginella sp. & Selaginellaceae & 1096 & 0.66 & 16.60 & 0.33 \\
\hline Siral $^{*}$ & Cucurbitaceae & 13 & 0.07 & 2.00 & 0.01 \\
\hline Solanum nigrum L. & Solanaceae & 178 & 0.92 & 1.93 & 0.18 \\
\hline
\end{tabular}


TABLE 5: Continued.

\begin{tabular}{|c|c|c|c|c|c|}
\hline Species & Family & Density (individuals ha ${ }^{-1}$ ) & Frequency (\%) & Abundance & IVI \\
\hline Solanum tuberosum L. & Solanaceae & 1202 & 0.46 & 26.00 & 0.32 \\
\hline Spilanthes paniculata Wall. ex DC. & Asteraceae & 10416 & 16.64 & 6.26 & 4.78 \\
\hline Spinacia oleracea L. & Chenopodiaceae & 852 & 1.25 & 6.79 & 0.37 \\
\hline Tagetes patula $\mathrm{L}$. & Asteraceae & 1466 & 3.76 & 3.89 & 0.89 \\
\hline Vigna sinensis (L.) Savi ex Hassk. & Papilionaceae & 218 & 0.86 & 2.54 & 0.18 \\
\hline Zingiber officinale Roscoe & Zingiberaceae & 1731 & 1.12 & 15.41 & 0.54 \\
\hline
\end{tabular}

${ }^{*}$ Locale name.

by $A$. malaccensis which is ranged from 17.94 to 72.63 with a mean of $49.36 \pm 2.64$. Dispersal limitation is an important ecological factor for controlling species distribution pattern [22]. Contagious distribution has been accepted as a characteristic pattern of plant occurrence in nature [23] and it is an indication of clusteredness. Contagious distribution of A. malaccensis is found in all study sites except in $\mathrm{KBG}$, Golaghat, where its distribution is random with abundance to frequency ratio of 0.04 . Contagious distribution of all the species irrespective of their habit in homegardens of northeast India is also reported by Sahoo et al. [24].

Ecological and socioeconomic factors including geographic location, climate, water availability, garden size and history, agricultural policy, market needs, food culture, and household preferences influence the species diversity of traditional homegardens [25-27]. Needs and interest of homegarden owners may also play a vital role in regulating floristic compositions of homegardens apart from edaphic, cultural, and socioeconomic factors [5]. Species richness provides an easily comprehensible expression of diversity which is affected by long term factors like community stability and evolutionary time as the heterogeneity of micro- and macroenvironments impact on the diversification of different communities [28]. Our investigation recorded 323 plant species (156 trees, 61 shrubs, and 106 herbs) indicating a high species richness of the study site. It is much higher than the earlier reports from Assam [29] as well as other parts of the world [30,31]. This may be due to the diverse cultural practices of the region and prevailing microclimatic conditions which provide suitable growing conditions for different plant species. Constituent of high floristic diversity is perhaps the potential of homegardens to serve as repositories of genetic diversity [5]. Very high species richness at all study sites is recorded in the present study ranging from 63 to 188 species with a mean of $113 \pm 7$ which is much higher than the earlier report from other parts of the world $[25,32]$. Although tree density $(1,400$ to 13,418 individuals $\mathrm{ha}^{-1}$ ) in different study sites was much higher than the recorded tree density in homegardens of other parts of India $[29,31]$, the basal cover of tree species $\left(0.54\right.$ to $\left.4.40 \mathrm{~m}^{2} \mathrm{ha}^{-1}\right)$ was much lesser than earlier report from Assam [29]. This may be due to the dominance of narrow range diameter class species in the study sites of upper Assam, northeast India.

Higher species richness indices (3.53 to 10.54) and species evenness indices ( 0.77 to 0.93 ) represent the floristic richness of all the study sites of upper Assam, northeast India. Shannon-Wiener diversity index is generally high for tropical forests of Indian subcontinent and ranged from 0.81 to 4.1 [33-36]. Shannon-Wiener diversity indices ranging from 2.52 to 3.78 represent the same structure of study sites of upper Assam and tropical forests of Indian subcontinent. Differences in species diversity between communities generally resulted from variations in site specificity [37]. Moreover, it is often correlated with rainfall and nutrient status of the site [38]. Although species diversity was high, the high similarity indices $(34.25 \%$ to $75.38 \%$ ) among different study sites indicate that floristic composition is characteristically similar in the region, may be because of common cultural interest of household owners. High diversity and low concentration dominance in study sites may be due to different levels of anthropogenic pressure in different sites.

\section{Conclusions}

The widespread cultivation of $A$. malaccensis in upper Assam, northeast India, offers a potential ex situ reservoir for the future conservation and management of this threatened tree. However, genetic diversity of such rare species of forest origin may be affected in managed ecosystems due to limited gene flow, inbreeding, and selection pressure. A thorough investigation of the genetic variation of the A. malaccensis cultivations investigated here is needed before their potential value to the ex situ conservation of this species can be confirmed.

\section{Conflict of Interests}

The authors (Dr. P. Saikia and Professor M. L. Khan) declare that there is no conflict of interests regarding the publication of this paper.

\section{Acknowledgments}

The authors would like to express their sincere gratitude towards the people of upper Assam, for the free access to their homegardens, for their cooperation during the survey and interviews, and for the permission to collect plant samples. Financial support from DBT, Govt. of India, through the project "Mapping and quantitative assessment of geographic distribution and population status of plant resources of Eastern Himalayan region" is highly acknowledged. 


\section{References}

[1] Anonymous, "Review of Significant Trade Aquilaria malaccensis," http://www.cites.org/sites/default/files/eng/com/pc/14/ E-PC14-09-02-02-A2.pdf.

[2] CITES, "Resolution," in Proceedings of the 9th Meeting of the Conference of the Parties (COP '94), Fort Lauderdale, Fla, USA, November 1994.

[3] IUCN, Asian Regional Workshop (Conservation \& Sustainable Management of Trees, Viet Nam) 1998, Aquilaria malaccensis. In: IUCN 2009, IUCN Red List of Threatened Species, Version 2009.2, 2010, http://www.iucnredlist.org.

[4] P. Saikia and M. L. Khan, "Agar (Aquilaria malaccensis Lam.): a promising crop in the homegardens of Upper Assam, Northeastern India," Journal of Tropical Agriculture, vol. 50, pp. 8-14, 2011.

[5] P. Saikia, B. I. Choudhury, and M. L. Khan, "Floristic composition and plant utilization pattern in homegardens of Upper Assam, India," Tropical Ecology, vol. 53, no. 1, pp. 105-118, 2012.

[6] P. Saikia and M. L. Khan, “Aquilaria malaccensis Lam., a Redlisted and highly exploited tree species in the Assamese home garden," Current Science, vol. 102, no. 4, pp. 546-547, 2012.

[7] S. C. Nath and N. Saikia, "Indigenous knowledge on utility and utilitarian aspects of Aquilaria malaccensis Lamk. in northeast India," Indian Journal of Traditional Knowledge, vol. 1, no. 1, pp. 47-58, 2002.

[8] N. Parthasarathy, "Tree diversity and distribution in undisturbed and human-impacted sites of tropical wet evergreen forest in southern Western Ghats, India," Biodiversity and Conservation, vol. 8, no. 10, pp. 1365-1381, 1999.

[9] H. Padalia, N. Chauhan, M. C. Porwal, and P. S. Roy, "Phytosociological observations on tree species diversity of Andaman Islands, India," Current Science, vol. 87, no. 6, pp. 799-806, 2004.

[10] Census of India, Census Report, Ministry of Home Affairs, Government of India, 2001.

[11] P. Saikia, Population structure and conservation practices of Aquilaria malaccensis Lam. in homegardens of upper Assam and its prospects in socio-economic development [Ph.D. thesis], Department of Forestry, NERIST (Deemed University), Arunachal Pradesh, India, 2011.

[12] R. Misra, Ecology Workbook, Oxford \& IBH Publishing Company, Calcutta, India, 1968.

[13] A. F. Magurran, Ecological Diversity and Its Measurement, Princeton University Press, Princeton, NJ, USA, 1988.

[14] C. E. Shannon and W. Wiener, The Mathematical Theory of Communities, University of Illinois Press, Urbana, Ill, USA, 1963.

[15] E. H. Simpson, "Measurement of diversity," Nature, vol. 163, article 688, 1949.

[16] T. Sørenson, "A method of establishing groups of equal amplitude on similarity of species content," Biologiske Skrifter K. Danske Videnskbernes Selskab, vol. 5, pp. 1-34, 1948.

[17] W. G. Whitford, "Distribution of woodland plants in relation to succession and clonal growth," Ecology, vol. 30, no. 2, pp. 199208, 1949.

[18] J. T. Curtis and G. Cottam, Plant Ecology Work Book, Laboratory Field Reference Manual, Burgess, Minneapolis, Minn, USA, 1956.

[19] J. E. Gordon and A. C. Newton, "Efficient floristic inventory for the assessment of tropical tree diversity: a comparative test of four alternative approaches," Forest Ecology and Management, vol. 237, no. 1-3, pp. 564-573, 2006.

[20] D. Jose, "Structure and productivity of the homegardens of Kerala: a case study," in Proceeding of the 4th Kerala Science Congress, C. G. R. Nair, Ed., pp. 17-19, Science, Technology and Environment Department, Government of Kerala, Thiruvananthapuram, India, 1992.

[21] E. P. Odum, Ecology, Holt, Reinhart and Winston, New York, NY, USA, 2005.

[22] S. P. Hubbell, R. B. Foster, S. T. O'Brien et al., "Light-gap disturbances, recruitment limitation, and tree diversity in a neotropical forest," Science, vol. 283, no. 5401, pp. 554-557, 1999.

[23] H. T. Odum, Fundamentals of Ecology, W.B. Saunders, Phiadelphia, Pa, USA, 1971.

[24] U. K. Sahoo, P. Rocky, K. Vanlalhriatpuia, and K. Upadhyaya, "Structural diversity and functional dynamism of traditional home gardens of North-East India," The Bioscan, vol. 1, pp. 159171,2010 .

[25] L. N. Trinh, J. W. Watson, N. N. Hue et al., "Agrobiodiversity conservation and development in Vietnamese home gardens," Agriculture, Ecosystems and Environment, vol. 97, no. 1-3, pp. 317-344, 2003.

[26] K. Kehlenbeck and B. L. Maass, "Crop diversity and classification of homegardens in Central Sulawesi, Indonesia," Agroforestry Systems, vol. 63, no. 1, pp. 53-62, 2005.

[27] O. T. Coomes and N. Ban, "Cultivated plant species diversity in home gardens of an amazonian peasant village in Northeastern Peru," Economic Botany, vol. 58, no. 3, pp. 420-434, 2004.

[28] R. K. Verma, K. S. Kapoor, S. P. Subramani, and R. S. Rawat, "Evaluation of plant diversity and soil quality under plantation raised in surface mined areas," Indian Journal of Forestry, vol. 27, no. 2, pp. 227-233, 2004.

[29] T. Das and A. K. Das, "Inventorying plant biodiversity in homegardens: a case study in Barak Valley, Assam, North East India," Current Science, vol. 89, no. 1, pp. 155-163, 2005.

[30] C. Padoch and W. de Jong, "The house gardens of Santa Rosa: diversity and variability in an amazonian agricultural system," Economic Botany, vol. 45, no. 2, pp. 166-175, 1991.

[31] B. M. Kumar, S. J. George, and S. Chinnamani, "Diversity, structure and standing stock of wood in the homegardens of Kerala in peninsular India," Agroforestry Systems, vol. 25, no. 3, pp. 243262, 1994.

[32] Z. L. Dao, W. S. Chen, H. J. Guo, H. L. Duan, and J. G. Duan, "Household based agrobiodiversity assessment (HH-ABA) of house garden in Hanlong village of Baosha, east slope of Gaoligong Mountains," Acta Botanica Yunnanica, supplement 12, pp. 102-112, 2000 (Chinese).

[33] P. Bhuyan, M. L. Khan, and R. S. Tripathi, "Tree diversity and population structure in undisturbed and human-impacted stands of tropical wet evergreen forest in Arunachal Pradesh, Eastern Himalayas, India," Biodiversity and Conservation, vol. 12, no. 8, pp. 1753-1773, 2003.

[34] N. Parthasarathy, V. Kinhal, and L. P. Kumar, "Plant species diversity and human impacts in the tropical wet evergreen forests of southern Western Ghats," in Proceedings of the IndoFrench Workshop on Tropical Forest Ecosystems: Natural Functioning and Anthropogenic Impact, pp. 26-27, French Institute, Puducherry, India, November 1992.

[35] J. S. Singh, Y. S. Rawat, and O. P. Chaturvedi, "Replacement of oak forest with pine in the Himalaya affects the nitrogen cycle," Nature, vol. 311, no. 5981, pp. 54-56, 1984. 
[36] N. Visalakshi, "Vegetation analysis of two tropical dry evergreen forests in southern India," Tropical Ecology, vol. 36, no. 1, pp. 117127, 1995.

[37] J. S. Denslow, "Gap partitioning among tropical rainforest trees," Biotropica, vol. 12, no. 2, pp. 47-55, 1980.

[38] G. S. Hartshorn, "Neotropical forest dynamics," Biotropica, vol. 12, no. 2, supplement, pp. 23-30, 1980. 

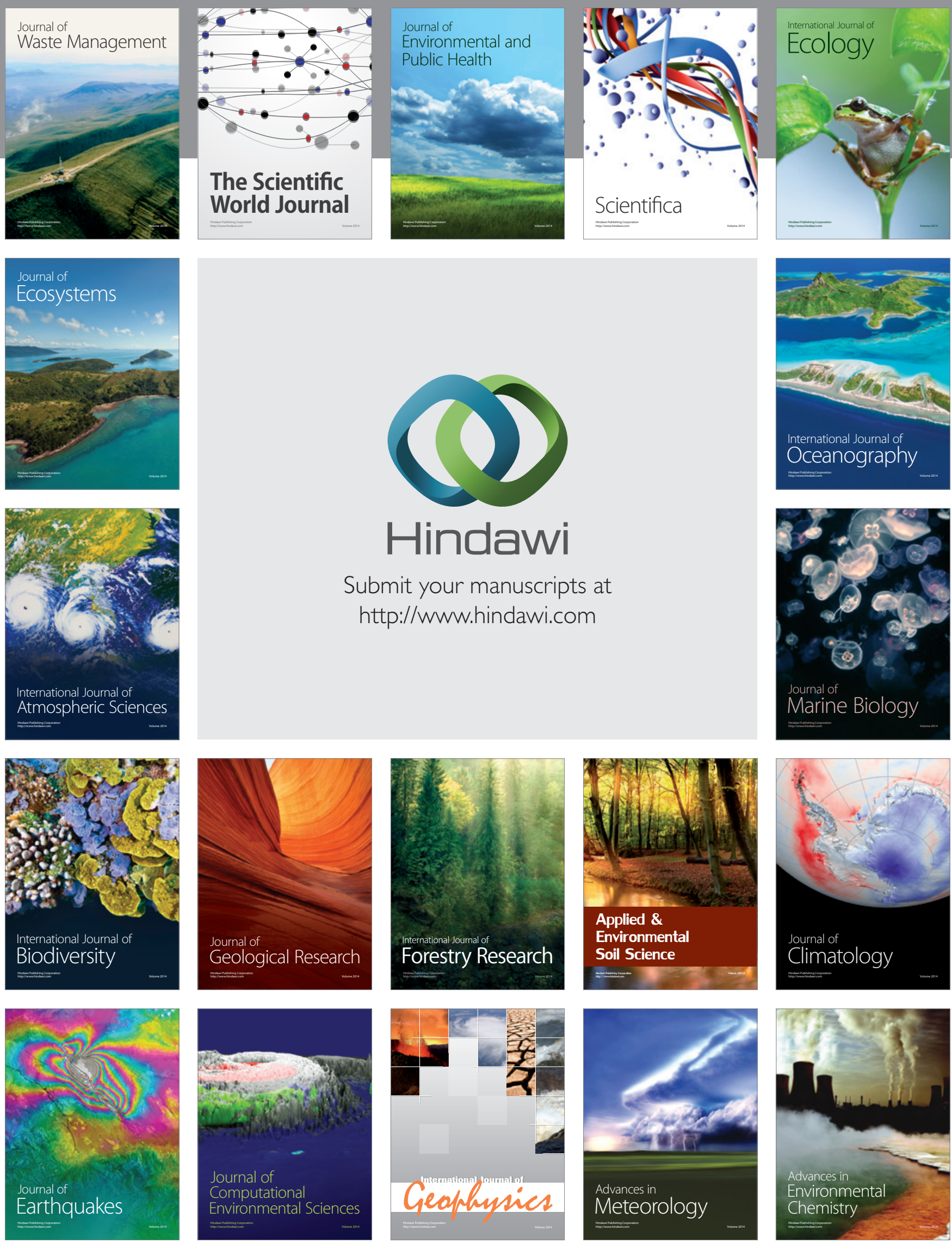